\title{
Comportement D'une Variété Précoce De Mil (Pennisetum glaucum (L.) R. Br) En Réponse A Différents Types De Fumures Combinées A Une Sécheresse De Fin De Cycle Au Nord De La Côte d'Ivoire
}

Laopé Ambroise Casimir Siene,

Université Péléforo Gon Coulibaly,

Département De Biologie Végétale, Côte d'Ivoire

Roger Bayala, Université Jean Lorougnon Guede, UFR Agroforesterie, Côte d'Ivoire

Mariame Conde, Baguehonon Diane Gofri, Ange Fabrice Béra Kouadio, Tâh Valentin Félix Nguettia, Université Péléforo Gon Coulibaly, Département De Biologie Végétale, Côte d'Ivoire

Doi:10.19044/esj.2020.v16n18p300 URL:http://dx.doi.org/10.19044/esj.2020.v16n18p300

\section{Résumé}

Cet article analyse l'influence de l'utilisation des fumures organiques et minérales sur la productivité du mil (Pennisetum glaucum (L.) R. Br) face à la baisse de la fertilité des sols dans un contexte de changements climatiques au Nord de la Côte d'Ivoire. Une étude a été menée pour analyser le comportement d'une variété précoce de mil en fonction de trois fumures organiques et une fumure minérale en situation de déficit hydrique post-floral. Le dispositif utilisé a été un bloc de Fisher complètement randomisé comportant quatre répétitions et cinq traitements (T1: témoin, T2: fiente de volaille, T3: déjection de lapin, T4: déjection de bœuf, T5: fumure minérale N-P-K-S-B+Urée). Les observations et mesures ont porté sur les stades de développement et les paramètres de croissance des plants. L'évapotranspiration réelle de la culture, le taux de satisfaction des besoins en eau des plants, le rendement et ses composantes ont été calculés ainsi que l'efficacité des fumures utilisées. Les résultats ont montré un déficit hydrique au cours de la phase reproductive. La variété de mil étudiée a présenté un cycle de développement plus court, une meilleure croissance, des valeurs de 
composantes du rendement élevées avec l'application de la fiente de volaille. La fumure fiente de volaille a été donc plus efficace que les autres fumures malgré la poche de sécheresse observée en fin de cycle. L'apport de la fiente de volaille peut être recommandé pour les cultures qui ont besoin de fertilisants, comme les variétés précoces de mil, car elle raccourcit la durée du cycle de culture permettant ainsi aux plants d'échapper aux conséquences néfastes du déficit hydrique de fin de cycle.

Mot clés : Déficit Hydrique, Fumures, Mil (Pennisetum Glaucum (L.) R. Br), Paramètres De Croissance, Phénologie, Rendement

\title{
Behaviour of an Early Variety of Millet (Pennisetum glaucum (L.) R. Br) in Response to Different Types of Manure Combined with Drought at the end of the Crop Cycle in Northern Côte d'Ivoire
}

\author{
Laopé Ambroise Casimir Siene, \\ Université Péléforo Gon Coulibaly, \\ Département De Biologie Végétale, Côte d'Ivoire \\ Roger Bayala, \\ Université Jean Lorougnon Guede, UFR Agroforesterie, Côte d'Ivoire \\ Mariame Conde, \\ Baguehonon Diane Gofri, \\ Ange Fabrice Béra Kouadio, \\ Tâh Valentin Félix Nguettia, \\ Université Péléforo Gon Coulibaly, \\ Département De Biologie Végétale, Côte d'Ivoire
}

\begin{abstract}
This Article Analyzes The Influence Of Organic And Mineral Manures On Millet (Pennisetum Glaucum (L.) R. Br.) Productivity In The Context Of Climate Change And Soil Fertility Degradation In Northern Côte d'Ivoire. To Do This, A Study Was Conducted To Analyze The Response Of An Early Variety Of Millet Based On Three Organic Manures And One Mineral Manure In Post-Floral Water Deficit Situations. The Experiment Had A Completely Randomized Block In Four Repetitions And Five Treatments (T1: Control, T2: Chicken Dropping; T3: Rabbit Dropping, T4: Cow Dung; T5: N-P-K-S-
\end{abstract}


B+Urea Mineral Fertilizer). Observations And Measurements Focused On The Stages Of Development And Plant Growth Parameters. The Crop Evapotranspiration, The Satisfaction Rate Of Plant's Water Needs, Yield And Components Were Calculated, And The Efficiency Of The Manure Used. The Results Showed A Water Deficit During The Reproductive Phase. The Millet Presented A Shorter Development Cycle, Better Growth And High Yield Component Values With Chicken Dropping Treatment. The Chicken Dropping Was More Effective Than Other Manure, Despite The Drought Observed At The End Of Crop Cycle. Applying Chicken Dropping May Be Recommended For Crops Needs Immediate Fertilizers, Such As Early Millet Varieties, Because It Shortens The Duration Of The Growing Cycle, Thus Allowing Plants To Escape The Harmful Consequences Of The End Of Crop Cycle Water Deficit.

Keywords: Water Deficit, Manure, Millet (Pennisetum Glaucum (L.) R. Br), Growth Parameters, Phenology, Yield

\section{Introduction}

Le mil (Pennisetum glaucum (L.) R.Br) est une céréale originaire d'Afrique de l'Ouest (Tostain, 1998). La production mondiale de mil était estimée à 28459020 tonnes sur une superficie de 31244420 ha avec un rendement moyen de $910 \mathrm{~kg} / \mathrm{ha}(\mathrm{FAO}, 2017)$. En Côte d'Ivoire, la production annuelle du mil est estimée à 60000 tonnes par an (Béninga et al., 2015). Le mil occupe la troisième place des céréales produites et consommées dans le pays, après le riz et le maïs. Avec son climat peu humide, ainsi que sa végétation du type herbeux, la partie Nord de la Côte d'Ivoire est la zone la plus propice à la culture du mil (Parry, 1982). La vaste majorité des variétés qui y sont cultivées sont traditionnelles (Sangaré et al., 2009).

Les rendements moyens sont de $500 \mathrm{~kg} / \mathrm{ha}$ en milieu paysan (Béninga, 2007). Cette faible production est due aux difficultés rencontrées par les producteurs dans la mise en place de la culture du mil. Ces difficultés sont essentiellement le faite d'attaques des ravageurs et des maladies, la non maîtrise des techniques de culture, la faible fertilité des sols, le coût élevé des intrants (Béninga, 2014). A ces problèmes s'ajoutent ceux causés par le changement climatique et ses conséquences à savoir la variabilité des pluies dans le temps et dans l'espace, l'incertitude dans le démarrage de la saison des pluies, et la modification des dates de semis (Sivakumar, 1988 ; Traoré et al., 2000 ; Bougma et al., 2018). Ainsi donc, avec le raccourcissement des saisons de pluies et les faibles précipitations, les variétés traditionnelles n'arriveraient plus à terminer leur cycle (Béninga et al., 2011). Comparativement aux variétés traditionnelles qui sont des mils tardifs, les variétés précoces ont des besoins en eaux moins importants que ceux des variétés traditionnelles 
(Moumouni, 2014). Ainsi, l'utilisation des variétés précoces serait une alternative dans la culture du mil face au raccourcissement de la durée des saisons de pluies (Béninga et al., 2011 ; Nguettia, 2013) d'une part et d'autre part, l'utilisation des fumures pourrait répondre aux problèmes de faible fertilité des sols. En effet, des études menées en vue d'augmenter le niveau de fertilité des sols et le rendement des cultures en général et du mil en particulier, ont été largement signalées dans la littérature (Zeinabou et al., 2014 ; Akanza et al, 2016 ; Somda et al., 2017 ; Ndiaye et al., 2019). Des travaux menés par Yang et Zhang (2006) et Aslani et Mehrvar (2012) ont montré que les semis tardifs des céréales souvent occasionnés par le décalage des dates de semis, en conditions de déficit hydrique et de fortes températures en phase de remplissage des grains provoquent une augmentation de la proportion des grains de petite taille et immatures.

Pour faire face à cette situation, le recours à des techniques agricoles permettant d'améliorer le niveau de fertilité du sol, tout en garantissant un niveau de production élevé du mil en cas de faibles précipitations, observées au cours du cycle cultural, notamment pendant les phases de remplissage et de maturation des graines s'impose. Il s'est agi dans cette étude de déterminer l'efficacité des fumures utilisées sur la productivité de la variété de mil étudiée en cas de pauses pluviométriques post-florale. Les objectifs spécifiques de cette étude ont été de (i) caractériser la phénologie de la variété de mil étudiée, (ii) déterminer l'évapotranspiration réelle ainsi que le taux de satisfaction des besoins en eau des plants, et (iii) comparer l'effet des fumures utilisées sur les paramètres de croissance et de productivité de cette variété.

\section{Matériel et méthodes}

\subsection{Site d'étude}

L'essai a été conduit sur le site expérimental de l'Université Péléforo Gon Coulibaly située dans le Département de Korhogo (9³4' de latitude Nord, $-5^{\circ} 37^{\prime}$ de longitude Ouest et $360 \mathrm{~m}$ d'altitude). Le climat est de type tropical sub-humide. II se caractérise par une saison sèche qui s'étend de novembre à mai et une saison de pluies de juin à octobre. Les mois les plus pluvieux sont juillet, août et septembre. La pluviométrie annuelle varie entre 1000 et $1600 \mathrm{~mm}$ (Diomandé, 2002). La température annuelle varie entre 24 ${ }^{\circ} \mathrm{C}$ et $36{ }^{\circ} \mathrm{C}$. Le sol de cette région est dominé par le type ferralsol (Perraud, 1971). Le sol du site expérimental est du type sablo-limoneux et les teneurs en C et N sont respectivement de 0,44 \% et 0,039 \% (Siéné et al., 2020).

\subsection{Matériel végétal}

Le matériel végétal utilisé est un cultivar de mil en provenance du Sénégal qui est caractérisée par sa précocité (cycle est d'environ 90 jours). Son 
rendement moyen varie de 2 à 2,5 t/ha rendement. Cette variété est peu résistante aux maladies et aux parasites (Anonyme, 2012).

\subsection{Dispositif expérimental et traitement étudiés}

Le dispositif expérimental a été en blocs de Fisher complètement randomisés avec quatre répétitions. Le facteur fertilisation a été constitué de cinq niveaux ou traitements à savoir : T1 (témoin : sans apport de fumure), T2 (apport de la fiente de volaille), T3 (apport de déjection de lapin), T4 (apport de déjection de bœuf) et T5 (apport d'engrais minéral de type NPKSB+Urée). Les teneurs en $\mathrm{C}$ et $\mathrm{N}$ des déjections sont données dans le tableau 1. Le dispositif expérimental a comporté un total de 20 parcelles élémentaires. Celles-ci de dimensions $2 \mathrm{~m}$ x 2,5 m ont été constituées de quatre billons cloisonnés. Chaque billon a comporté cinq poquets, soit un total de vingt poquets par parcelle élémentaire. Les écartements ont été de 0,5 m entre deux poquets de la même ligne et $0,8 \mathrm{~m}$ entre deux billons successifs. Les parcelles élémentaires du même bloc ont été séparées d'une distance de $1 \mathrm{~m}$ et les blocs ont été séparés d'une distance de $1 \mathrm{~m}$.

\subsection{Conduite de la culture}

Le semis a été effectué sur billons cloisonnés en humide le 16 août 2018. Le semis a été tardif car il s'effectue en fin juillet ou en début août (Anonyme, 2005). Cette date a été choisie de sorte à faire coïncider le début de la phase reproductive avec la fin de la saison des pluies. Les fumures organiques ont été apportées à la dose de 20 t/ha (Chaibou, 2013); soit 10 $\mathrm{kg} /$ parcelle élémentaire. Cette dose a été divisée par quatre en tenant compte du nombre de billons contenus dans les parcelles élémentaires. Ainsi, la dose de $2,5 \mathrm{~kg}$ de fumure organique a été apportée par billon. Ces apports ont été faits quinze jours avant le semis. Le premier apport d'engrais minéral a été fait au démariage en utilisant du NPKSB (15-15-15+6S+1B), à la dose de 4,46 g/poquet; soit une dose de 89,2 g/parcelle élémentaire, à raison de 20 poquets par parcelle élémentaire. Cette dose a été apportée au poquet à l'aide d'une capsule à une distance de $5 \mathrm{~cm}$ du plant. Le deuxième apport a été fait à la montaison sous forme d'urée (46-00-00) à une dose de 7,86 g/poquet, soit deux capsules par trou à $5 \mathrm{~cm}$ du plant. La dose de 157,2 g/parcelle élémentaire a été apportée. Le démariage a été effectué $15 \mathrm{JAS}$, à raison de trois plants par poquet.

\subsection{Mesures et observations}

\subsubsection{Observations phénologiques et mesures agro-morphologiques}

Les mesures et observations ont porté sur la phénologie (le temps d'émergence, la durée de la phase végétative, la durée de la phase reproductive et la durée du cycle), sur les paramètres morphologiques (la hauteur du plant, 
le nombre de talles par poquet, le nombre de feuilles vertes par poquet, le nombre total de feuilles par poquet) ainsi que sur le rendement et ses composantes (le nombre d'épis par poquet, le nombre de grains par épi, et le poids de 1000 grains). L’efficacité des fumures a été déterminée par rapport au témoin à l'aide de la relation suivante :

$$
E(\%)=\frac{R d t F-R d t T}{R d t T} X 100
$$

Avec :

$\mathrm{E}$ : efficacité de la fumure $; \mathrm{RdtF}$ : rendement de la fumure $; \mathrm{RdtT}$ : rendement du témoin.

\subsubsection{Calcul des besoins en eau des plants et indice de sécheresse}

La distribution des pluies au cours du cycle cultural a été suivie à l'aide d'un pluviomètre à lecture directe installé sur le site expérimental. La consommation hydrique (ETR) et les besoins en eau du maïs ont été calculés par le logiciel Cropwat version 8.0 (FAO, 2002) à partir des données journalières de températures maximum et minimum $\left({ }^{\circ} \mathrm{C}\right)$, $1^{\prime}$ 'humidité relative $(\%)$, la vitesse du vent $\left(\mathrm{m} \mathrm{s}^{-1}\right)$, la pluviométrie $(\mathrm{mm})$ et l'insolation (heures). La méthode de calcul de l'indice de sécheresse, [(ETM - ETR)/ETM], utilisée par Sarr et al., (2012), a été utilisée pour caractériser l'intensité du déficit hydrique subi par les plants tout au long du cycle de la culture.

Avec :

- $\quad \mathrm{ETM}=$ évapotranspiration maximale ; et

- $\quad$ ETR = évapotranspiration réelle.

\subsection{Analyses statistiques des données}

Les analyses de variance ont été effectuées à l'aide du logiciel Statistix 8.1 pour mettre en évidence l'effet de la fumure sur la croissance et le rendement du mil. La comparaison des moyennes a été effectuée avec le test de Tukey HSD au seuil de 5\%.

\section{Résultats}

\subsection{Evapotranspiration réelle et taux de satisfaction des besoins en eau} des plants

Au cours du cycle cultural, une quantité totale de $369,9 \mathrm{~mm}$ a été enregistrée (tableau 2). Une pause pluviométrique a été observée entre le

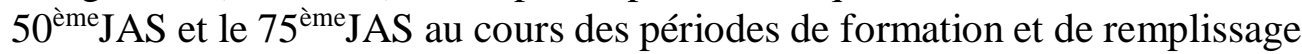

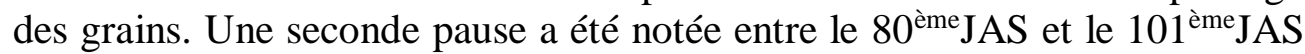
au cours de la maturation des grains (figure 1).

Durant l'expérimentation, les quantités de pluies reçues au cours des phases végétative et reproductive ainsi que le cumul des valeurs 
d'évapotranspiration réelle des plants (ETR) et de taux de satisfaction des besoins en eau (TSATcycle) correspondantes ont été calculés (tableau 2). Au cours de la phase végétative la quantité de pluie enregistrée a été de 297,5 mm. Le calcul de l'ETR montre que les plants témoins (T1) ont eu la plus forte consommation hydrique de $361,6 \mathrm{~mm}$. Tandis que, les plants fertilisés avec la fiente de volaille (T2), ont présenté une plus faible consommation en eau de $312,67 \mathrm{~mm}$. Durant la phase reproductive, une quantité de pluie de $72,4 \mathrm{~mm}$ a été enregistrée. Les plants ayant reçu la fiente de volaille (T2) ont eu une consommation en eau faible égale à $154,50 \mathrm{~mm}$. L'analyse des résultats des taux de satisfaction des besoins en eau montre que les plants n'ont pas subi de contrainte hydrique durant la phase végétative avec des valeurs qui ont varié entre 82 et $95 \%$. Par contre, pendant la phase reproductive, les plants de tous les traitements ont subi une contrainte hydrique sévère avec des taux de satisfaction en eau inferieurs à $50 \%$. Cependant, en considérant tout le cycle de la culture, les résultats montrent que les plants ayant reçu la fiente de volaille (T2) ont eu une bonne alimentation hydrique avec un taux de satisfaction de $80 \%$. Les plants des autres traitements ont par contre subi une contrainte hydrique avec des taux de satisfaction qui ont varié entre $70 \%$ et $72 \%$. La valeur de ce paramètre permet d'apprécier l'intensité de la contrainte hydrique subie par la culture. Ainsi, avec des valeurs de taux de satisfaction en eau de $70 \%, 71 \%$ et $72 \%$, à l'échelle du cycle de la culture, les plants sont en situation d'un stress hydrique modéré. En effet, en phase de reproductive, les taux de satisfaction en eau ont varié entre 44 et $47 \%$ soit des indices de sécheresse de 0,53 et 0,56 .

\subsection{Caractérisation de la phénologie}

Au cours de cette étude, les durées des phases phénologiques du mil ont été calculées (tableau 3). L'analyse de variance a montré une différence aussi bien au niveau de la durée de la phase végétative que de la durée du cycle de culture. Les plants ayant reçu la fiente de volaille ont réalisé une durée de phase végétative plus courte (soit 54 jours) par rapport à ceux des autres traitements. Les parcelles ayant reçu la fiente de volaille ont eu une durée de cycle de culture plus courte ( 82 jours). Soit une différence de 12 jours par rapport au témoin, de 9 jours par rapport à la déjection de lapin et de 11 pour le bœuf et l'engrais minéral NPKSB+Urée.

\subsection{Effet des fumures sur l'évolution des paramètres de croissance 2.3.1 Hauteur totale des plants}

Au cours du cycle culturale, les valeurs de hauteur totale des plants ont été mesurées en fonction des traitements (figure 2). Ces valeurs ont augmenté au cours du cycle quel que soit le traitement. L'analyse de variance a montré une différence significative entre les plants des différents traitements 
appliqués. Les plants ayant reçu la fiente de volaille ont présenté les valeurs de hauteur totale les plus élevées en fin de cycle avec une moyenne de 118,67 $\mathrm{cm}$. La déjection de bœuf quant à elle, a présenté la plus faible valeur de hauteur avec une moyenne de 93,92 cm. Concernant les plants du traitement témoin et ceux ayant reçu l'engrais minéral NPKSB+Urée (T5), et la déjection de lapin (T3), les valeurs moyennes de hauteur totale ont été respectivement de $106,08 \mathrm{~cm} ; 112,17 \mathrm{~cm}$ et $104,83 \mathrm{~cm}$.

\subsubsection{Nombre total de talles par poquet}

Le nombre maximal de talles par poquet (figure 3) a été obtenu à la floraison. Les résultats de l'analyse statistique ont montré une différence significative entre les différents traitements. Les plants traités avec la fiente de volaille (T2) ont produit un plus grand nombre de talles $(13,42)$, suivi des plants ayant reçu l'engrais minéral NPKSB+Urée (T5), la déjection de bœuf (T4) et les plants témoins (T1). Le plus faible nombre de talles a été obtenu au niveau des plants ayant reçu la déjection de lapin (T3).

\subsubsection{Nombre total de feuilles par poquet}

En fin de cycle cultural, le nombre total de feuilles produites a été compté en fonction des traitements (figure 4). L'analyse de variance a montré une différence significative entre les différents traitements. Les plants ayant reçu l'engrais minéral NPKSB+Urée (T5) ont produit le plus grand nombre de feuilles suivis de ceux qui ont été fertilisés avec la fiente de volaille (T2) avec respectivement une valeur moyenne de 75 feuilles et 72 feuilles. Par contre, les plants témoins (T1) ont produit le plus faible nombre total de feuilles (50 feuilles).

\subsection{Efficacité des fumures étudiées sur le rendement et ses composantes}

Les valeurs du rendement et de ses composantes ont été calculées après la récolte (tableau 4). L'analyse statistique a montré une différence significative entre les traitements au niveau des composantes du rendement (tableau 4). Les plants fertilisés avec la fiente de volaille ont obtenu les meilleures valeurs moyennes de nombre d'épi par poquet $(3,67)$ et de nombre moyen de grain par épis $(17643,6)$. Le poids de 1000 grains a été plus faible au niveau des plants fertilisés avec la déjection de bœuf comparés autres traitements. En ce qui concerne le rendement, la fiente de volaille (T2) a permis d'obtenir un rendement plus élevé $(1,80 \mathrm{t} / \mathrm{ha})$ que les autres traitements.

Les fertilisants utilisés ont présenté des effets différents par rapport au témoin (tableau 4). Le pourcentage d'efficacité obtenu avec la fumure fiente de volaille par rapport au témoin a été de 164,25 \% suivi de l'engrais minéral 
NPKSB+Urée avec 57,44\%. Par contre les fumures déjections de bœuf et de lapin ont été moins efficace par rapport au témoin avec respectivement des pourcentages de $-10,40 \%$ et $-20,67 \%$.

\section{Discussion}

Concernant la phénologie, l'apport de la fiente de volaille a raccourci la durée du cycle des plants l'ayant reçu. Son action pourrait s'expliquer par les nutriments qu'elle contient et leurs assimilations par la plante. Cette différence d'effet entre la fiente de volaille et les autres types de fumures utilisées dans notre étude pourrait s'expliquer par la vitesse de minéralisation des amendements organiques. En effet, les constituants des amendements organiques ne sont pas directement disponibles, ils doivent d'abord être minéralisés (Asdrubal et al., 2006 et Segnou et al., 2012). Et, selon Etter (2017), le fumier de volaille et les farines animales sont rapidement minéralisés alors que le fumier de bovins nécessite un temps important. De plus, la minéralisation de la fiente de volaille favoriserait une disponibilité en azote totale et en phosphore assimilable dans le sol (Dean et al., 2000). Ce phosphore serait un facteur de précocité selon Kati (2012) et pourrait expliquer le raccourcissement de la phase végétative.

Notre analyse a aussi été axée sur l'effet des fumures sur l'évolution des paramètres de croissance. Les plants ayant reçu la fiente de volaille ont présenté les meilleures valeurs de paramètre de croissance au cours du cycle. Cela pourrait être dû à la quantité importante d'azote contenue dans cette fumure qui est disponible pour les plants pendant leur phase de croissance. En effet, la fiente de volaille utilisée dans notre étude a présenté une teneur en azote élevé (3\%) comparée aux autres fumures organiques. Ce résultat corrobore ceux de Ndour et al. (2016) et Equiterre (2009) qui ont montré que la fiente de volaille est à peu près six fois plus riche en azote que celui de bovin. De même, Siboukeur (2013) a montré que la fiente de volaille est beaucoup plus riche en éléments fertilisants comparée aux autres fumiers organiques.

En rapport avec le rendement, la meilleure valeur a été obtenue avec la fiente de volaille. Il a été de 1,80 t/ha, proche du rendement moyen obtenu avec les variétés de mil précoces du Sénégal qui varie entre 2 et 2,5 t/ha (Anonyme, 2012). Le faible rendement obtenu avec la déjection de bœuf et de lapin pourrait s'expliquer par leur faible vitesse de minéralisation. Les déjections de bœuf et de lapin utilisées présentent des rapports $\mathrm{C} / \mathrm{N}$ respectivement de 20 et 24,5 qui traduisent potentiellement de faibles vitesses de minéralisation, comparées à la fiente de volaille dont le rapport $\mathrm{C} / \mathrm{N}$ est 13 . En effet, le rapport $\mathrm{C} / \mathrm{N}$ d'une matière renseigne sur sa vitesse de minéralisation et la faible valeur de ce rapport pourrait traduire la relative rapide minéralisation de l'azote et par conséquent sa disponibilité (Aké et al., 
2018). Cette faible vitesse de minéralisation n'a pas permis de mettre à la disponibilité des plants les éléments nutritifs nécessaires à leur productivité. Selon Chabalier et al. (2006), la fiente de volaille, plus riche en $\mathrm{N}, \mathrm{P}_{2} \mathrm{O}_{5}$ et $\mathrm{K}_{2} \mathrm{O}$ agirait comme un engrais minéral en apportant peu d'humus aux plants. La faible vitesse de minéralisation observée chez les fumures déjection de lapin et bœuf couplée au déficit hydrique post-floral n'a pas permis aux plants d'assurer une bonne synthèse d'assimilât nécessaire au bon développement des épis et au bon remplissage des grains. Ainsi, les plants de mil fertilisés avec ces fumures ont obtenu un rendement inférieur à celui du témoin. Ces résultats sont en accord avec ceux obtenus par Siéné et al. (2016) qui ont montré que le déficit hydrique enregistré au cours de la phase de remplissage des grains se traduit par une forte réduction du rendement des plants en conditions de fertilisation azotée quel que soit le régime hydrique. En effet, une répartition irrégulière des pluies a été observée au cours du cycle de culture dans notre étude. Un stress hydrique sévère a été observé pendant la phase reproductive. Or au cours de cette phase, un déficit hydrique a des conséquences immédiates sur les épis se trouvant encore en phase précoce d'évolution (épiaison, floraison et fécondation), ou sur les grains en début de remplissage (Siéné et al., 2016).

Par contre, la fiente de volaille épandue sur les billons cloisonnés, en raccourcissant la durée du cycle des plants, leur a permis d'échapper à l'effet du déficit hydrique post-floral enregistrée dans notre étude. Ce qui a permis d'obtenir un résultat contraire à ceux obtenus par Yang et Zhang (2006) et Aslani et Mehrvar (2012) qui ont montré qu'avec un semis tardif des céréales en conditions de déficit hydrique et de fortes températures en phase de remplissage des grains, il y aurait une augmentation de la proportion des grains de petite taille et immatures. A vu du comportement des plants de la variété de mil étudiée en présence des fumures utilisées, l'apport de la fiente de volaille peut être recommandé pour les cultures qui ont besoin dans l'immédiat de fertilisants, comme les variétés précoces de mil, car cela augmente la teneur des éléments majeurs, la capacité d'échange cationique (CEC) et améliore la qualité physico-chimique et biologique du sol (Adeleye et al., 2010).

\section{Conclusion}

Cette étude a montré le rôle joué par les fumures notamment la fiente de volaille sur la productivité du mil en cas de faibles précipitations enregistrées au cours du cycle de culture. Un déficit hydrique post-floral a été enregistré au cours de notre étude. La durée du cycle de culture a été plus courte au niveau des plants ayant reçu la fiente de volaille. Concernant les paramètres de croissance, les valeurs les plus élevées ont été obtenues avec les plants fertilisés avec la fiente de volaille. Le rendement et ses composantes ont été meilleurs avec la fiente de volaille à cause de sa minéralisation rapide et sa 
richesse en éléments nutritifs par rapport aux autres types de fumures. De plus, la fiente de volaille grâce à son facteur de précocité a permis aux plants d'échapper à l'effet du stress post-floral enregistrée dans notre étude. Malgré donc ce déficit hydrique enregistré en fin de cycle, l'on a pu obtenir un rendement avec cette fumure proche du rendement moyen des variétés de mil précoce du Sénégal. La fiente de volaille pourrait donc être recommandé pour les cultures à besoin immédiat de fertilisants, comme les variétés précoces de mil en cas de sécheresse post-florale, car elle raccourci la durée du cycle de culture, permettant ainsi aux plants d'échapper aux conséquences néfastes du déficit hydrique de fin de cycle.

\section{Conflit d'intérêts}

Les auteurs déclarent sur l'honneur l'absence de tout conflit d'intérêt.

\section{Contributions des auteurs}

Laopé Ambroise Casimir SIENE a rédigé le protocole d'expérimentation, contribué à la mise en place de l'essai, à la vérification et à l'exploitation des données (analyses statistiques) ainsi qu'à la rédaction de la première version du manuscrit. Roger BAYALA et Mariame CONDE ont veillé à la conformité des résultats et contribué à la rédaction du manuscrit. Baguehonon Diane GOFRI, Ange Fabrice Béra KOUADIO et Tâh Valentin Félix NGUETTIA ont participé à la mise en place de l'essai, au suivi et à la collecte des données.

\section{Remerciements}

Nos remerciements vont à l'endroit de Dr TUO Yalamoussa Docteur en entomologie agricole à l'Université Péléforo Gon Coulibaly pour l'aide qu'il nous a apportée lors des traitements phytosanitaires des parcelles. Nos remerciements vont également à l'endroit de Monsieur Moussa Dione du service technique de l'Ecole Nationale Supérieure d'Agriculture (ThièsSénégal) pour la fourniture de semence de mil et à travers lui tous les autres agents dudit service.

Tableau 1 : Résultats Analytiques Des 3 Echantillons De Fumure Organique.

\begin{tabular}{ccccc}
\hline Nature Echantillon & Carbone (\%) & M.O (\%) & N (\%) & C/N \\
\hline Déjection De Bœuf & 42,98 & 74,11 & 2,19 & 20,00 \\
Dejection De Lapin & 36,43 & 62,81 & 1,48 & 24,50 \\
Fiente De Poulet & 38,19 & 65,83 & 3,01 & 13,00 \\
\hline
\end{tabular}

Tableau 2 : Quantité de pluie reçue, évapotranspiration réelle et taux de satisfaction des besoins en eau des plants en fonction des phases phénologiques et des traitements.

\begin{tabular}{clllllllll}
\hline & \multicolumn{3}{c}{ Phase végétative } & \multicolumn{3}{c}{ Phase reproductive } & \multicolumn{3}{c}{ Cycle de la culture } \\
\cline { 2 - 8 } Traitements & Pluie & ETR & TSAT & Pluie & ETR & TSAT & Pluie & ETR & TSAT \\
& $(\mathrm{mm})$ & $(\mathrm{mm})$ & $(\%)$ & $(\mathrm{mm})$ & $(\mathrm{mm})$ & $(\%)$ & $(\mathrm{mm})$ & $(\mathrm{mm})$ & cycle
\end{tabular}




\begin{tabular}{llllllllll}
\hline T1 & 297,5 & 361,60 & 82,00 & 72,4 & 164,20 & 44,00 & 369,9 & 525,80 & 70 \\
T2 & 297,5 & 312,67 & 95,00 & 72,4 & 154,50 & 47,00 & 369,9 & 467,17 & 80 \\
T3 & 297,5 & 350,71 & 85,00 & 72,4 & 161,91 & 45,00 & 369,9 & 512,62 & 72 \\
T4 & 297,5 & 356,10 & 83,00 & 72,4 & 165,29 & 44,00 & 369,9 & 521,39 & 71 \\
T5 & 297,5 & 356,10 & 84,00 & 72,4 & 165,29 & 44,00 & 369,9 & 521,39 & 71 \\
\hline
\end{tabular}

$\mathrm{T} 1:$ témoin sans apport d'engrais ; T2 : fiente de poulet ; T3 déjection de lapin : T4 : déjection de bœuf; T5 : Engrais minéral N-P-K-S-B+Urée, Evapotranspiration réelle cumulée (ETR), taux moyen de satisfaction des besoins en eau sur le cycle (TSATcycle), TSAT durant les phases phénologiques. World-Weather-Online (https://www.historiquemeteo.net/afrique/cote-d-ivoire/korhogo/).

Tableau 3 : Les phases phénologiques du mil en fonction des traitements.

\begin{tabular}{ccccc}
\hline Traitements & $\begin{array}{c}\text { Emergence } \\
\text { (Jours) }\end{array}$ & $\begin{array}{c}\text { Phase } \\
\text { végétative } \\
\text { (Jours) }\end{array}$ & $\begin{array}{c}\text { Phase } \\
\text { reproductive } \\
\text { (Jours) }\end{array}$ & $\begin{array}{c}\text { Durée du cycle } \\
\text { (Jours) }\end{array}$ \\
\hline T1 & $3^{\mathrm{a}}$ & $63^{\mathrm{a}}$ & $31^{\mathrm{a}}$ & $94^{\mathrm{a}}$ \\
T2 & $3^{\mathrm{a}}$ & $54^{\mathrm{b}}$ & $28^{\mathrm{a}}$ & $82^{\mathrm{b}}$ \\
T3 & $3^{\mathrm{a}}$ & $61^{\mathrm{a}}$ & $30^{\mathrm{a}}$ & $91^{\mathrm{a}}$ \\
T4 & $3^{\mathrm{a}}$ & $62^{\mathrm{a}}$ & $31^{\mathrm{a}}$ & $93^{\mathrm{a}}$ \\
T5 & $3^{\mathrm{a}}$ & $62^{\mathrm{a}}$ & $31^{\mathrm{a}}$ & $93^{\mathrm{a}}$ \\
\hline
\end{tabular}

$\mathrm{T} 1$ : témoin sans apport d'engrais ; T2 : fiente de poulet ; T3 déjection de lapin : T4 :

déjection de bœuf; T5 : Engrais minéral N-P-K-S-B+Urée. Pour une variable et un effet donné, les moyennes affectées de la même lettre ne sont pas significativement différentes au seuil de $5 \%$, test de Tukey (HSD).

Tableau 4 : Rendement, composantes du rendement et efficacité des fumures utilisées sur le rendement par rapport au témoin.

\begin{tabular}{cccccc}
\hline Traitements & $\begin{array}{c}\text { Nombre } \\
\text { d'épis/poquet }\end{array}$ & $\begin{array}{c}\text { Nombre de } \\
\text { grains/épis }\end{array}$ & PMG $(\mathrm{g})$ & $\begin{array}{c}\text { Rendement } \\
\text { en t/ha }\end{array}$ & $\begin{array}{c}\text { Efficacité } \\
(\%)\end{array}$ \\
\hline T1 & $1,89^{\mathrm{b}}$ & $2985,2^{\mathrm{b}}$ & $9,16^{\mathrm{a}}$ & $0,68^{\mathrm{b}}$ & - \\
T2 & $3,67^{\mathrm{a}}$ & $17643,6^{\mathrm{a}}$ & $8,92^{\mathrm{a}}$ & $1,80^{\mathrm{a}}$ & 164,25 \\
T3 & $1,84^{\mathrm{b}}$ & $4173,7^{\mathrm{b}}$ & $8,74^{\mathrm{a}}$ & $0,54^{\mathrm{b}}$ & $-20,67$ \\
T4 & $2,11^{\mathrm{b}}$ & $4434,3^{\mathrm{b}}$ & $7,62^{\mathrm{b}}$ & $0,61^{\mathrm{b}}$ & $-10,40$ \\
T5 & $2,62^{\mathrm{ab}}$ & $8209,9^{\mathrm{b}}$ & $9,02^{\mathrm{a}}$ & $1,07^{\mathrm{b}}$ & 57,44 \\
\hline
\end{tabular}

T1 : témoin sans apport d'engrais ; T2 : fiente de poulet ; T3 déjection de lapin : T4 : déjection de bœuf; T5 : Engrais minéral N-P-K-S-B+Urée. PMG : poids de 1000 grains. Pour une variable et un effet donné, les moyennes affectées de la même lettre ne sont pas significativement différentes au seuil de $5 \%$, test de Tukey (HSD). 


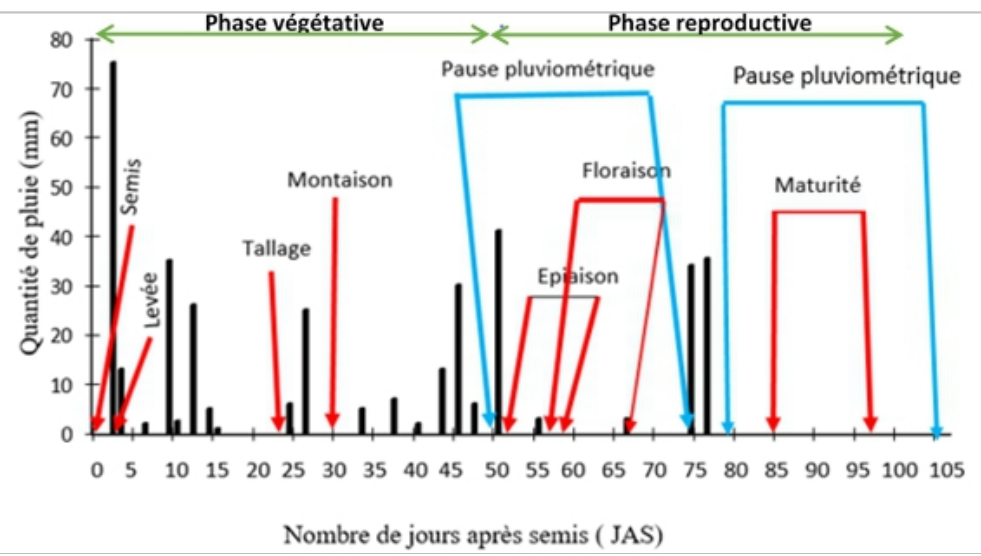

Figure 1 : Répartition des pluies journalières au cours de l'expérimentation.

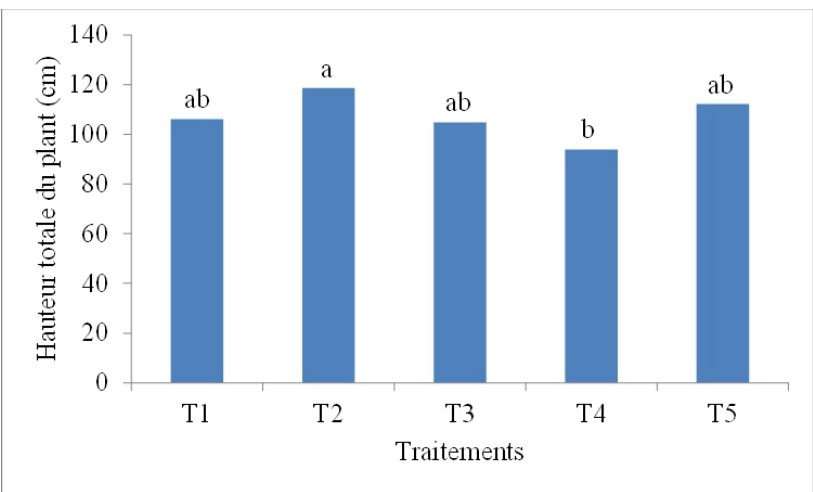

Figure 2 : Hauteur totale des plants par poquet en fonction des traitements.

T1 : témoin sans apport d'engrais; T2 : fiente de poulet ; T3 déjection de lapin : T4 : déjection de bœuf; T5 : Engrais minéral N-P-K-S-B+Urée. Pour une variable et un effet donné, les moyennes affectées de la même lettre ne sont pas significativement différentes au seuil de $5 \%$, test de Tukey (HSD).

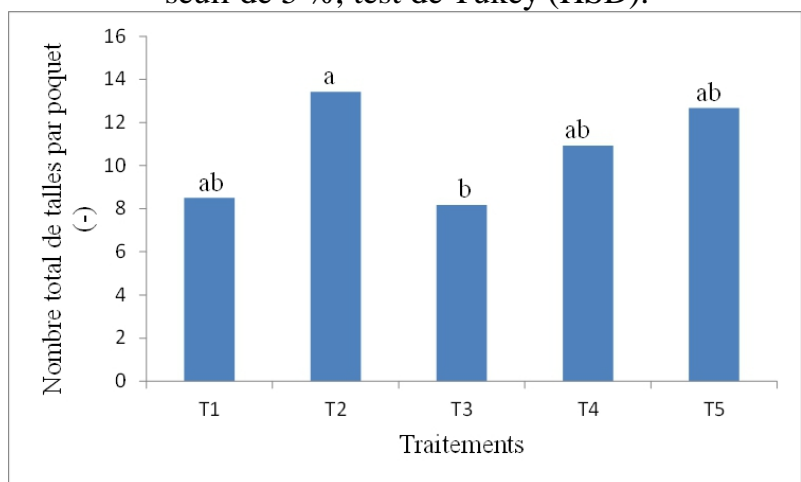

Figure 3 : Nombre maximal de talles par poquet en fonction des traitements.

T1 : témoin sans apport d'engrais ; T2 : fiente de poulet ; T3 déjection de lapin : T4 : déjection de bœuf; T5 : Engrais minéral N-P-K-S-B+Urée. Pour une variable et un effet donné, les moyennes affectées de la même lettre ne sont pas significativement différentes au seuil de $5 \%$, test de Tukey (HSD). 


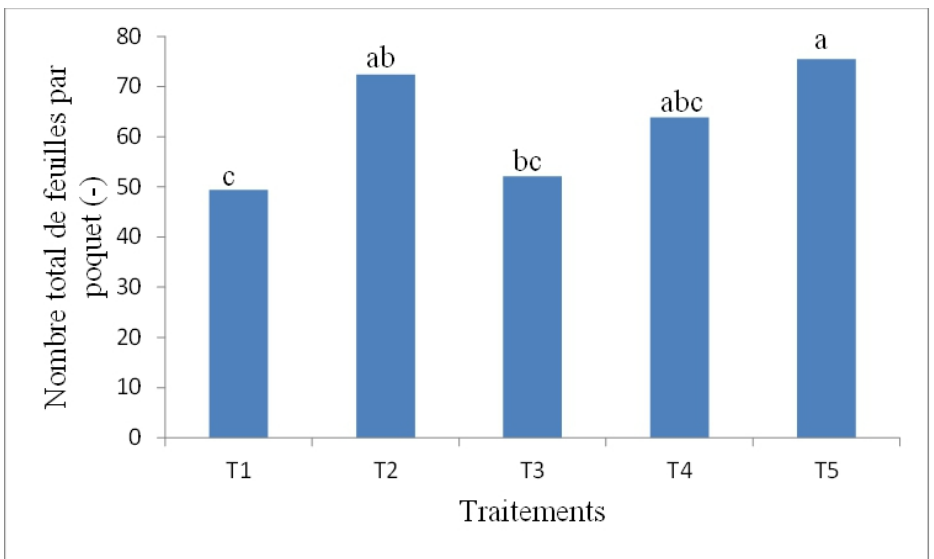

Figure 4 : Nombre total de feuilles par poquet en fonction des traitements.

T1 : témoin sans apport d'engrais ; T2 : fiente de poulet ; T3 déjection de lapin : T4 : déjection de bœuf; T5 : Engrais minéral N-P-K-S-B+Urée. Pour une variable et un effet donné, les moyennes affectées de la même lettre ne sont pas significativement différentes au seuil de $5 \%$, test de Tukey (HSD).

\section{References:}

1. Adeleye E.O., Ayeni L.S., and Ojeniyi S.O., 2010. Effect of poultry manure on soil physico-chemical properties, leaf nutrient contents and yield of yam (Dioscorea rotundata) on Alfisol in Southwestern Nigeria. J. Am. Sci., 6(10):871-878.

2. Akanza K.P., Sanogo S., et N'Da H.A., 2016. Influence combinée des fumures organique et minérale sur la nutrition et le rendement du maïs : impact sur le diagnostic des carences du sol. Tropicultura, 34(2) : 208-220.

3. Aké H.T.B., Bi Tra T., et Dogbo O.D., 2018. Caractéristiques physico-chimiques des composts à base de sous-produits de ferme de Songon en Côte d'Ivoire. Int. J. Biol. Chem. Sci., 12(1): 596-609.

4. Anonyme, 2005. Centre National de Recherche Agronomique, CNRA. Bien cultiver le mil en Côte d'Ivoire.

5. Anonyme, 2012. Ministère du développement rural et de l'hydraulique. Catalogue variétale.

6. Asdrubal M., Sylvie D., Charonnat C., Denys F., Fresse J.C., et Thomas J .M., 2006. Fertilisation et amendements. Educagri éditions, 131 pages.

7. Aslani F., and Mehrvar M.R., 2012. Responses of wheat genotypes as affected by different sowing date. Asian Journal of Agricultural Sciences, 4(1): 72-74.

8. Béninga M.B., 2007. Génétique, amélioration et vulgarisation du mil (Pennisetum glaucum (L.) R.Br) en Côte d'Ivoire. Thèse de Doctorat 
d'Etat, UFR Biosciences, Université de Cocody, Abidjan, Côte d'Ivoire, 179p.

9. Béninga M.B., Sangaré A., N'Guettia S.P.A., et Coulibaly M.Y., 2011. Mise en place d'une collection de ressources génétiques du mil (Pennisetum glaucum (L.) R. Br) en Côte d'Ivoire. Agronomie Africaine, 23(3) : 193 - 204.

10. Béninga M.B., 2014. Diagnostic des systèmes de culture à base de mil (Pennisetum glaucum (L.) R. Br) en Côte d'Ivoire et perspectives d'amélioration. Journal of Applied Biosciences, 79 : 6878-6886.

11. Béninga M.B., et Aclé D., 2015. Evaluation des pertes en grains de mil dues aux insectes. European Scientific Journal, 11(21) : 226-275.

12. Bougma L.A., Mahamadi H.O., Nerbewendé S., Mahamadou S., Didier B., et Ronnie V., 2018. Perception paysannes de l'impact du changement climatique sur le mil dans les zones sahélienne et soudano-sahélienne du Burkina Faso. Afrique Sciences, 14(4) : 264275.

13. Chabalier P.F., Van De Kerchove V., et Macary S.H., 2006. Guide de la fertilisation organique à la réunion. CIRAD. Chambre d'agriculture de la réunion. $304 \mathrm{p}$

14. Chaibou Z., 2013. Effet du phospho-compost sur la production du mil (Pennisetum glaucum (L.) R. Br). Mémoire pour l'obtention d'un Master de maîtrise en gestion intégrée de la fertilité des sols. Université Polytechnique de Bobo- Dioulasso, Burkina-Faso.47p.

15. Dean D.S., Earl C.S., and Raymond E.K., 2000. Irrigation management for corn in the Northern Great Plains, USA. Irrig. Sci., 19: 107-114.

16. Diomandé I., 2002. Les pesticides utilisés dans la production des mangues. Mémoire de Diplôme Universitaire de Technologie (DUT), option: gestion agro-pastorale, Université de Bouaké/URES de Korhogo, Côte d'Ivoire.

17. Equiterre, 2009. Les amendements organiques : fumiers et composts. Module 7, chapitre 12, 19 pages.

18. Etter A., 2017. Engrais organiques: rendement et qualité. Revue UFA 10/ 2017, 2 pages.

19. FAO, 2002. Crop Evapotranspiration Guidelines for Computing Crop Water Requirements: Guidelines for Computing Crop Water Requirements (FAO Irrigation and Drainage Paper No.56,).

20. FAO, 2017. http://faostat3.fao.org/download/Q/QC/F/ Consulté en mai 2018

21. Kati Y., 2012. Contribution à la maîtrise de la fertilisation phosphatée des sols de montagne: cas des sols de vergers de pommiers d'Ichemoul (Aures-Algérie). Mémoire pour l'obtention du Diplôme 
de Magister en Sciences Agronomiques, Université El-Hadj LakhdarBatna, Institut des Sciences Vétérinaires et des Sciences Agronomiques, Option : pédologie, p. 84.

22. Moumouni K.H., 2014. Construction d'une carte génétique pour le mil (Pennisetum glaucum (L.) R. Br) par une approche de génotypage par séquençage (GBS). Mémoire, Université de Laval de Québec, Québec, p. 111.

23. N'Guettia Y.R., 2013. Etude de vulnérabilité du secteur agricole face aux changements climatiques en Côte d'Ivoire. $105 \mathrm{p}$

24. Ndiaye A., Ndiaye O., Bamba B., Guèye M., et Sawané O., 2019. Effets de la fertilisation organo- minérale sur la croissance et le rendement du mil sanio (Pennisetum glaucum (L.) R. Br) en Haute Casamance (Sénégal). European Scientific Journal, 15(33) : 155-170.

25. N'Dour N.Y.B., N'Diénor M., Corfini N.A., Aubry C., Masse D., Paillat J., M., Diène J., Parnaudeau V., et Lounkara S., 2016. Recyclage des déchets organiques en agriculture. Éditions Ouée, 2016, 28 pages.

26. Parry G., 1982. Le cotonnier et ses produits collection « technique agricole et production végétale »; Paris France Maisonneuve et Larousse et ACCT, pp.30-404.

27. Perraud A., 1971. Les sols. In: Avenard J. M., Eldin E., Girard G., Sircoulon J., Touchebeuf P., Guillaumet J. L., Adjanohoun E. et Perraud A. (Eds.), Le milieu naturel de la Côte d'Ivoire. Éditions O.R.S.T.O.M., Paris, France, 50, pp. 269-391, 1971.

28. Sangaré A., Koffi E., Akamou F., et Fall C.A., 2009. Rapport sur l'état des ressources phytogénétiques pour l'agriculture et l'alimentation. $64 \mathrm{p}$.

29. Sarr, B., Atta, S., et Kafando L., 2012. Revue des indices climatiques utilisés dans les systèmes d'assurances agricoles indicielles en Afrique. Science et changements planétaires/Sécheresse, 23(4), 255260.

30. Segnou J., Akoa A., Youmbi E., et Njoya J., 2012. Effet de la fertilisation minérale et organique sur le rendement en fruits du piment (Capsicum annuum L.; solanaceae) en zone forestière de basse altitude au Cameroun. 10 pages.

31. Siboukeur A., 2013. Appréciation de la valeur fertilisante de différents types de fumier. Mémoire de fin d'études, département des Sciences Agronomiques, faculté des sciences de la nature et de la vie et sciences de la terre et de l'univers, Université kasdiMerbah Ouargla, 78 pages.

32. Siéné L.A.C., Dago F.S., Diouf O., et Aké S., 2016. Evaluation de l'impact de stress hydriques sur la croissance et le rendement du mil (Pennisetum glaucum (L.) R. Br) pour deux conditions de fertilisation 
azotée dans un environnement tropical semi-aride. International Journal of Innovation and Applied Studies, 14(3): 779-789.

33. Siéné L.A.C., Doumbouya M., Traoré M.S., Condé M., N'Guettia T.V.F., et Koné M., 2020. Effet de quatre types de fertilisants sur la croissance et la productivité de deux génotypes de maïs (Zea mays L.) en cas d'un semis tardif à Korhogo au Centre-Nord de la Côte d'Ivoire. Int. J. Biol. Chem. Sci., 14(1): 55-68.

34. Sivakumar, M.V.K., 1988. Predicting rainy season potential from the onset of rains in southern sahelian and soudanian climatic zones of West Africa. Agricultural and Forest Meteorology, 42: 295-305.

35. Somda B. B., Ouattara B., Serme I., Pouya M. B., Lompo F., Taonda S.J.B., et Sedogo P.M., 2017. Détermination des doses optimales de fumures organo-minérales en micro-dose dans la zone soudanosahélienne du Burkina Faso. International Journal of Biological and Chimical Sciences, 11(2): 670-683.

36. Tostain S., 1998. Le Mil, une longue histoire : hypothèses sur sa domestication et ses migrations. In : Plantes et paysages d'Afrique : une histoire à explorer, Chastanet M. Ed. Paris, France, KarthalaCRA, pp.461-490p.

37. Traoré, S., Reyniers, F., Vaksmann, M., Koné, B., Sidibé, A., Yoroté, A., Yattara, K. et Kouressy M., 2000. Adaptation à la sécheresse des écotypes locaux de sorghos du Mali. Sécheresse, 11: 227-237.

38. Yang J., and Zhang J., 2006. Grain filling of cereals under soil drying. New Phytologist, 169: 223-236.

39. Zeinabou H., Mahamane S., Bismarck N.H., Bado B.V., Lompo F. et André B., 2014. Effet de la combinaison des fumures organominérales et de la rotation niébé-mil sur la nutrition azotée et les rendements du mil au sahel. International Journal of Biological and Chimical Sciences, 8(4): 1620-1632. 